\title{
The past, present, and future of urological quality improvement collaboratives
}

\author{
Adam C. Reese ${ }^{1}$, Serge Ginzburg ${ }^{2}$ \\ ${ }^{1}$ Department of Urology, Lewis Katz School of Medicine, Temple University, PA, USA; ${ }^{2}$ Department of Urology, Albert Einstein Medical Center, \\ Philadelphia, PA, USA \\ Contributions: (I) Conception and design: AC Reese, S Ginzburg; (II) Administrative support: AC Reese, S Ginzburg; (III) Provision of study material \\ or patients: AC Reese, S Ginzburg; (IV) Collection and assembly of data: AC Reese, S Ginzburg; (V) Data analysis and interpretation: AC Reese, \\ S Ginzburg; (VI) Manuscript writing: All authors; (VII) Final approval of manuscript: All authors. \\ Correspondence to: Serge Ginzburg, MD. Director, Urologic Oncology, Department of Urology, Albert Einstein Medical Center, Moss/Sley Building, \\ 3rd Floor, 1200 Tabor Road, Philadelphia, PA 19141, USA.. Email: ginzburs@einstein.edu.
}

\begin{abstract}
Surgical quality improvement collaboratives (QIC) have been established across the nation in numerous specialties. These QICs have shown efficacy in improving the quality, safety and value of care delivered to patients with a wide range of medical conditions. In recent years, urological QICs have emerged, including regional collaboratives such as the Michigan Urological Surgical Improvement Collaborative (MUSIC) and Pennsylvania Urologic Regional Collaborative (PURC), as well as the national American Urological Association Quality Registry Program (AQUA). These urological collaboratives, developed with an initial focus on prostate cancer, have demonstrated an ability to accurately measure prostate cancer outcomes, compare these outcomes among providers and institutions, and enact change among both patients and providers to optimize outcomes for men with prostate cancer. Physician-led regional collaboratives may be uniquely positioned to respond quickly to the rapidly-evolving healthcare landscape and enact practice and provider-level changes when appropriate. This review describes the historical background, current structure and function, and potential future directions of these urologic QICs.
\end{abstract}

Keywords: Quality improvement collaborative (QIC); Pennsylvania Urologic Regional Collaborative (PURC); Michigan Urological Surgical Improvement Collaborative (MUSIC); urological collaborative

Submitted Apr 15, 2019. Accepted for publication Sep 27, 2019.

doi: $10.21037 /$ tau.2019.10.18

View this article at: http://dx.doi.org/10.21037/tau.2019.10.18

\section{Introduction}

Healthcare reform, with a focus on quality, has taken center stage as US healthcare expenditures surpass $\$ 3.5$ trillion in 2017, accounting for $17.5 \%$ of GDP (1). In this environment, quality improvement collaboratives (QIC) are proving to be an effective vehicle for change, but this was not always the case. Ineffective historical approaches to quality improvement such as top-down government policy directives or short-reaching local hospital- or practice-level efforts, fueled by imperfect claims-based public outcomes reporting, ultimately made way for the physician-led collaboratives $(2,3)$.
As early as 1914, Earnest Armory Codman with his "end result system" advocated for longitudinal data tracking and analysis to identify areas of improvement, but this was not widely adopted at the time (4). The formal directive came in 1999 with a publication "To Err is Human" from the Institute of Medicine (IOM), highlighting that healthcare lags more than a decade behind other high-risk industries in patient safety, and calling for a $50 \%$ reduction in medical errors over 5 years (5). IOM mandated public reporting of adverse events resulting in harm or death and encouraged voluntary public reporting of broader set of errors by individual providers and health systems-a common practice today that has been adopted by both private and 


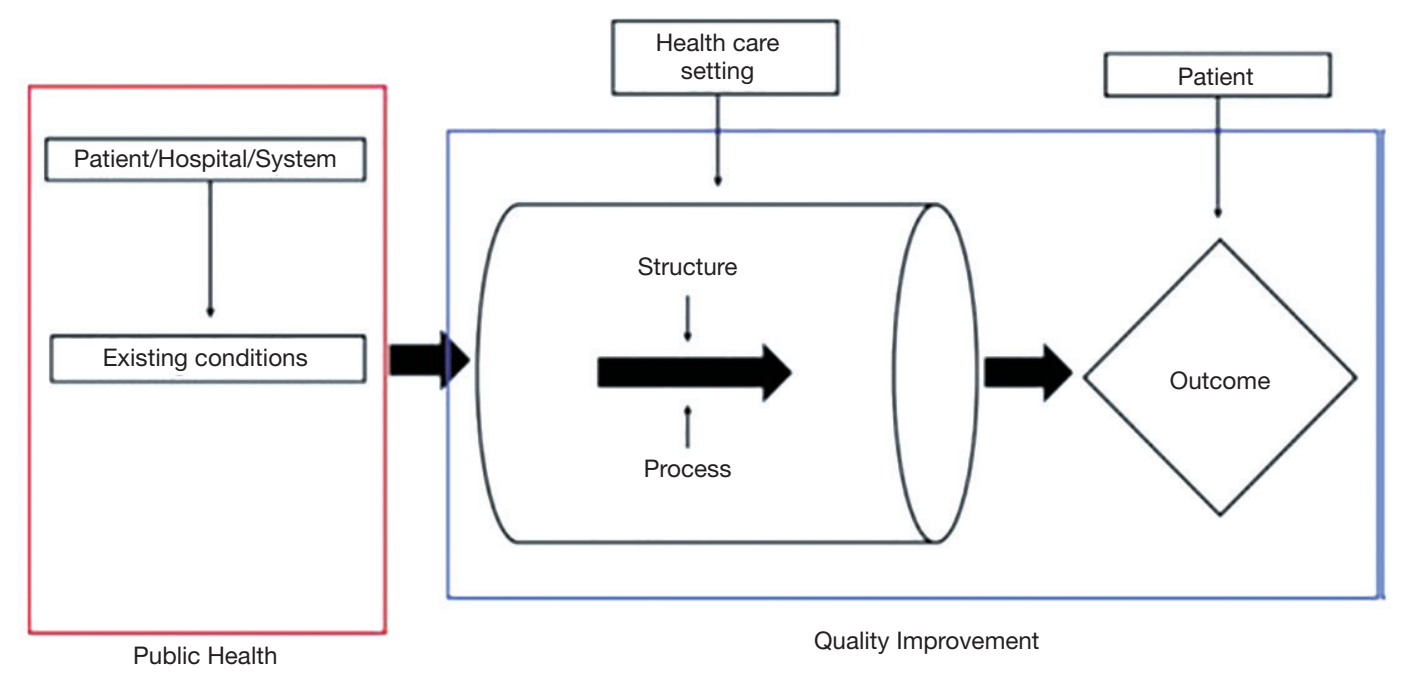

Figure 1 Donabedian conceptual model. The Donabedian model divides health care into three components: structure, process, and outcome. "Structure" refers to the infrastructure in which health care is delivered (e.g., hospital size, availability of specific resources, etc.). "Process" is the care actually delivered to the patient. "Outcome" refers to the end product of an episode of care (12).

public sectors $(6,7)$. National, state-wide or regional level quality initiatives emerged to address the gaps in quality, safety and value of delivered care. In this review we focus on evolution of surgical QICs in urology.

\section{What comprises a QIC}

A QIC is a group of contributors that is committed to the use of shared data to improve patient safety, quality, or value of care across participating sites. Such initiatives do not arise in a vacuum and require an environment ripe for change, with the catalyst often being large variation in the quality and cost of care. For many collaboratives, the initial focus is disease- or procedure-specific, but often expands to a wider scope of practice once the collaborative infrastructure is in place.

Critically important to the success of a QIC is the physician leader, often with national or international recognition, who creates a culture of trust and can recruit a core team of physician champions that will coalesce around common goals to improve quality, safety and value (8).

QICs require transparent, high-fidelity, and standardized data collection with regular auditing (9). At a minimum, each participating site contributes an actively engaged physician champion and a trained data abstractor. The coordinating center typically includes a physician leader, an epidemiologist/statistician, a data analyst, a data auditor, a quality improvement nurse and administrative staff. The coordinating center manages data and audits, provides high-level analytic support, facilitates collaborative sessions, provides meaningful feedback and plays a central role in the success of the collaborative. Shared data are anonymized to promote collaboration among individual providers and practices, rather than competition. Participants review collaborative-wide, practice-level, or provider-level data to identify areas of opportunity, implement change, and measure impact using internal and external benchmarks. The results of interventions are distributed to participants to enact change at the individual or practice level.

There are several conceptual models that have been designed to assess quality, with the Donabedian component model currently preferred by many authors (9-11). Avedis Donabedian proposed that quality of care can be distilled into three components-structure, process and outcomes (Figure 1) (12). Structure is a relatively fixed component, referring to one's practice setting, hospital size and type, equipment, and individual surgeons and their patient volume. Process is the manner in which the care is delivered. This component is typically the most actionable and thus a perfect target for QICs. Outcomes are the product of care, such as morbidity and mortality as well as clinical, and financial metrics. While these metrics may be the easiest to ascertain, QICs can help shift focus to new or more meaningful metrics, such as patient-reported outcomes.

QICs are perhaps most effective on a regional level 
to assure an abundance of data yet allow geographical proximity to engage the providers through in-person collaborative sessions and meaningful benchmarking. Additionally, regional scale may allow for focus on granular details, flexibility in innovation, commitment to proposed interventions, and faster implementation of directives (8).

\section{History of surgical QICs}

The Northern New England Cardiovascular Disease Study Group was a pioneering surgical QIC, initiated in 1987 and focused on understanding outcomes of coronary artery bypass grafting (13). The collaborative was created in response to public reporting of only partially risk-adjusted surgical outcomes, lacking detailed risk-adjustments for patient, hospital, provider and surgical techniques among others (2). This collaborative was a voluntary consortium of providers, researchers and hospital administrators from 5 hospitals in neighboring states, with a common vision to establish a clinical registry and improve patient outcomes.

Subsequently, the National Veterans Affairs Surgical Risk Study (NVASRS) was created in 1991 in response to public scrutiny of patient safety and quality of care in the VA system. This initiative focused on optimizing 30-day post-operative outcomes among patients undergoing major surgical procedures at the VA. In 1994, due to the success of this effort, the program was converted into the ongoing National Surgical Quality Improvement Program (NSQIP), and in 2001 the American College of Surgeons adopted NSQIP for the private sector $(14,15)$. ACS-NSQIP data are obtained directly from the medical chart and metrics are risk-adjusted for patient characteristics and hospital case mix. In its current form, ACS-NSQIP is a pay-to-participate initiative where participants provide their own clinical reviewer. The program reports valuable data, including observed and expected risk-adjusted outcomes, that can then translate into local quality improvement initiatives. Since acting on this data is not required to participate, ACS-NSQIP serves a function similar to a robust data registry with valuable feedback and benchmarking potential, although its effectiveness on outcomes was recently called into question (16).

The Michigan Surgical Improvement Collaborative (MSQC) was established in 2004 with an initial focus on major general and vascular surgical procedures. MSQC is funded by a dominant private insurer in that state-Blue Cross and Blue Shield Michigan (BCBSM)/Blue Care Network (17). A unique state-wide Value Partnership,
MSQC is hospital-based rather than practice-based and is comprised of a diverse mix of community and academic institutions where members were initially incentivized to participate and collaborate, rather than compensated based on performance (18). BCBSM did not coordinate the collaborative, nor could it identify individual hospital or provider data, but could only see it in aggregate. Feedback to the participants was provided quarterly by the coordinating center, with in-person collaborative sessions and idea exchange. The fundamental quality improvement process focused on identifying best performers and their best practices, and then disseminating this information to all participants (8). Over time, pay-for-performance elements were introduced by assigning individual hospitals scores based on degree of participation and engagement, improvement in quality, clinical process and outcomes (17). Hospitals were organized into peer groups by size and location, with differing incentives for large and medium-sized hospitals as compared to small and rural hospitals, and in 2019, BCBSM Hospital Payfor-Performance Program will pay an additional $5 \%$ of statewide inpatient and outpatient operating payments\$190 million statewide. (ref: 2019 P4P program document, https://www.bcbsm.com/content/dam/public/Providers/ Documents/value/2019-hospital-pay-performanceprogram.pdf accessed on 8/12/19) Expansion of payorfunded QICs in other medical and surgical areas in Michigan continued, and at the time of this publication at least 17 unique QIC projects are active across the state.

\section{Urologic quality initiatives}

\section{Michigan Urological Surgery Improvement Collaborative (MUSIC)}

Inspired by the success of established QIC in other medical specialties, three academic and private practices in Michigan formed the Urologic Surgery Quality Collaborative (USQC) in 2009. Over the two-year existence of USQC, with minimal financial support, the collaborative was able to expand to seven practices and was successful in characterizing the use of imaging in low risk prostate cancer and the use of intravesical therapies in the management of bladder cancer across the region $(19,20)$.

Following the initial success of the USQC collaborative, additional financial support through a Value Partnership with BCBSM allowed for the creation of the larger MUSIC in 2011. MUSIC was established with the goals of 
collecting clinically credible data, comparing performance among peers, sharing best practices, and implementing change in clinical behavior in order to achieve more efficient utilization of healthcare resources, improve care delivery, and enhance the quality, value, and outcomes of treatment provided to men in Michigan with prostate cancer (21). Structured in a similar way to MSQC, each individual practice within MUSIC designates a clinical champion responsible for representing their practice, ensuring accurate collection of clinical data, and reporting findings from the collaborative back to the providers within their practice. A centralized coordinating center is responsible for recruiting and onboarding practices, auditing and maintaining the data, providing administrative and analytical support, and organizing periodic meetings to share data and coordinate future directions. MUSIC leverages its affiliation with Health Services Research powerhouse, the University of Michigan, for analytics and statistical support. Since its establishment, 45 independent urology practices have joined MUSIC, representing over $90 \%$ of practicing urologists in the state of Michigan (22).

Prostate cancer was chosen as the initial focus of the MUSIC collaborative, largely due to the high prevalence of this disease and large variation in care and cost within urology practices $(23,24)$. The MUSIC leadership team identified several aspects of prostate cancer care as their initial focus, including: the use of appropriate imaging for men with prostate cancer, decreasing complications due to prostate biopsy, improving outcomes of radical prostatectomy (RP), and encouraging shared decision making among providers and patients deciding on initial management strategy of localized prostate cancer. The following discussion will briefly review MUSIC's accomplishments toward each of these areas of focus.

\section{Use of appropriate imaging among men with prostate cancer}

Despite knowledge that metastases are rarely present in men with clinically localized prostate cancer, staging imaging studies are frequently ordered for such patients. These unnecessary imaging studies come with a significant cost to the healthcare system and can result in clinically insignificant incidental findings with resultant downstream ramifications. The problem of inappropriate imaging among men with prostate cancer was so important that the American Urological Association has included two statements regarding the use of appropriate imaging in its "Choosing Wisely" campaign (25).
The MUSIC collaborative has been successful in addressing this problem using a stepwise process, ultimately demonstrating an ability to increase the appropriate use of imaging for men with prostate cancer in Michigan. As an initial step, the collaborative identified clinical factors predictive of the presence of metastatic disease at the time of prostate cancer diagnosis $(26,27)$. Using these data, MUSIC was able to establish appropriateness criteria for the use of staging imaging in men with newly diagnosed prostate cancer. Subsequently these recommendations were disseminated along with educational materials to the individual urology practices and clinical champions were encouraged to discuss these recommendations with their colleagues. Ultimately, this intervention was found to decrease the inappropriate use of bone scans from $11.0 \%$ to $6.5 \%$, inappropriate use of CT scans from $14.7 \%$ to $7.7 \%$, and decreased imaging variability among practices $(28,29)$.

\section{Decreasing complications of prostate biopsy}

Prostate biopsy is one of the most common procedures performed by urologists in the United States. Although generally well tolerated, there is a significant risk of serious post-biopsy complications, typically due to biopsy-related infections (30,31). The collaborative efforts of MUSIC have been successful in decreasing the incidence of biopsyrelated complications in Michigan, primarily through the reduction of infectious complications.

MUSIC initially characterized the risk of hospitalization after prostate biopsy, and determined that most of these hospitalizations were due to infections with fluoroquinolone resistant organisms (31). Using these data, they were able to develop strategies to address the problem of fluoroquinolone resistance, namely the use of tailored antibiotics based on results of a rectal swab, or the use of augmented antibiotic therapy. Implication of these recommendations was subsequently shown to decrease the incidence of post-biopsy infection related hospitalizations by $53 \%(32)$.

\section{Improving outcomes of RP}

Oncologic outcomes, complication rates, and postoperative functional outcomes after RP have been shown to vary both among surgeons and among different urology practices $(33,34)$. To this end, MUSIC has taken several steps towards decreasing complications rates and improving and standardizing RP outcomes in Michigan.

During their quarterly collaborative-wide meeting, MUSIC holds breakout sessions for surgeons who perform 
RP aimed at improving surgical technique. These sessions focus on specific aspects of the operation, typically feature expert high-volume surgeons to lead the discussion, and allow for review of surgical videos and discussion among peers to facilitate learning. Furthermore, MUSIC offers a deidentified video review service, whereby a surgeon can submit a video of a robotic RP case that is then evaluated by their peers and rated for technical skill. The surgeon can then use this feedback to identify areas in need of technical improvement that hopefully should translate to improved post-operative outcomes for his or her patients.

MUSIC has also proposed objective criteria to define an uncomplicated recovery after RP, which can then be used to compare complications rates among surgeons and practices in a standardized fashion (35). Additionally, a platform has been developed that allows patients to self-report postoperative outcomes electronically. These patient-reported outcomes not only have the potential to improve care of individual patients by identifying the specific concerns of that patient, but can also be tabulated and compared among surgeons to identify patterns among individual surgeons that can then be targeted for performance improvement.

\section{Shared decision making in newly diagnosed prostate cancer}

Decisions regarding the optimal treatment modality in men with newly diagnosed prostate cancer are difficult, and must account for oncologic outcomes, patient preference, posttreatment functional outcomes, and costs to the healthcare system, among other factors. For many men with low risk tumors, active surveillance (AS) is an appealing management strategy as it offers the potential to minimize treatmentrelated morbidity without compromising cancer control. Despite the potential upside of AS, this approach has not been uniformly embraced, as demonstrated by data showing a wide variability in the use of AS among urology practices (36,37).

A potential source of variability in the practice of AS is the lack of objective criteria for identifying appropriate candidates for AS, determining how best to follow patients while on surveillance, and defining factors that should trigger a transition to definitive treatment. To eliminate uncertainty, MUSIC developed a roadmap to assist providers managing patients with low risk prostate cancer interested in AS $(36,38)$. This roadmap establishes discrete criteria that can be used to identify candidates for AS, accounting for a patient's ethnicity, life expectancy, prostate cancer risk parameters, and concerns regarding preservation of sexual function. Furthermore, the roadmap makes specific recommendations of how to follow patients while on surveillance and details factors that should potentially trigger a transition to more aggressive management. Interventions such as this roadmap will hopefully serve to encourage shared decision making among patients and providers, and decrease some of the variability in care among individual providers and urology practices.

\section{Pennsylvania Urologic Regional Collaborative (PURC)}

The PURC was established in 2015 as the first regional urology collaborative in the state of Pennsylvania. The collaborative was established with the goals of improving the diagnosis and care of men with prostate cancer in the Delaware Valley Region. PURC initially formed as a collaborative of 6 large academic and community-based urology practices in Philadelphia, but has since expanded to Western Pennsylvania and Southern New Jersey. Currently, PURC collects data from 132 providers managing men with prostate cancer across 11 practices. As of March 2019, PURC had collected data on over 10,500 men diagnosed with prostate cancer or undergoing prostate biopsy across the region.

The PURC structure is similar to that of MUSIC, with a central coordinating center and clinical physician champions designated to represent each practice site. Patient care data are manually abstracted from the electronic medical record by trained data abstractors and compiled in a centralized database. Regular audits are performed by the PURC coordinating center to ensure data accuracy.

The overall collaborative is subdivided into individual working groups, each tasked with investigating a specific aspect of prostate cancer care. Current working groups focus on prostate cancer imaging, reducing biopsy related complications, and encouraging and standardizing the use of AS for men with low risk prostate cancer.

The PURC collaborative has been successful in achieving a number of goals, including: the characterization of prostate cancer imaging patterns across the Delaware Valley region $(39,40)$, describing prophylactic antibiotic protocols prior to prostate biopsy (41), and characterizing variation in the use of AS among men with low risk prostate cancer $(37,42)$. Similar to MUSIC's success, through participating physician education PURC was able to significantly reduce inappropriate $\mathrm{CT}$ and Bone Scan utilization in men with low risk prostate cancer (SGunpublished data). 


\section{American Urological Association Quality Registry Program (AQUA)}

In 2014, the American Urological Association established the AUA Quality (AQUA) Registry, the first national registry for urologic disease in the United States $(43,44)$. AQUA was designed to measure, report, and improve healthcare quality and outcomes for patients with urologic disease. The initial focus of AQUA is improving outcomes for men with prostate cancer, although the registry aims to expand to other urological diseases in the future.

The AQUA registry has been recognized by the Centers for Medicare and Medicaid Services (CMS) as a Qualified Clinical Data Registry (QCDR), including Physician Quality Reporting System (PQRS) and non-PQRS measures for public reporting. Furthermore, the registry can be used for the Merit-Based Incentive Payment System (MIPS) whereby CMS encourages the reporting of quality data through financial incentives to urology practices.

Between 2014 and 2016, 125 practices and 1,148 urologists joined the AQUA registry (45). Over this same period of time, the registry collected data on 35,437 men with prostate cancer. Preliminary analysis of these data have identified important trends in prostate cancer across the United States, including a rising incidence of high risk disease over time, and the increased use of AS for men with low risk tumors (46). These preliminary data demonstrate the potential power of the AQUA registry given its ability to analyze a very large number of geographically disparate patient populations spanning the United States.

\section{Future directions}

A strength of QICs is their ability to adapt to a changing healthcare landscape and rapidly refocus their efforts on relevant initiatives. This capacity has been demonstrated by both PURC and MUSIC, as they have taken on new directions over time as the collaboratives evolved.

The use of genomic testing has been rapidly adopted by many physicians caring for men with prostate cancer. These relatively novel tests require ongoing investigation to characterize their efficacy in various stages of disease, compare their results to their competitors, and quantify their value while taking into account the costs of these tests. PURC is working to better characterize the role of genomic testing by establishing a genomic working group to evaluate patterns of testing across the collaborative. To date, the collaborative has found a wide variation in the overall use of genomic testing, in the specific tests utilized, and in the settings in which these test are ordered among providers (47).

Similarly, MUSIC has made great strides in objectively characterizing the utility of genomic testing with the Genomics in Michigan Impacting Observation or Radiation (G-MINOR) prospective randomized trial $(48,49)$. This trial aims to evaluate the role of genomic testing in deciding whether to proceed with adjuvant radiation therapy after $\mathrm{RP}$ in patients with high-risk pathologic features. G-MINOR randomizes patients after $\mathrm{RP}$ to either standard post-operative risk assessment based on clinicopathologic data (using the CAPRA-S score) or risk assessment using the CAPRA-S score plus the Decipher (GenomeDX) genomic test of RP tissue. The study aims to determine the role of the Decipher assay in influencing the decision of whether to proceed with adjuvant radiation therapy after RP.

Urologic QICs are also working to address the opioid epidemic that is currently sweeping the United States (50). PURC has established a working group to develop and disseminate an opioid-sparing post-operative pathway for men undergoing RP. Similarly, MUSIC is addressing this issue by distributing materials focused on patient and physician education of opioid-sparing protocols, specifically addressing RP pain management best-practices (James M. Dupree, MD, personal communication 2018).

Following the success and rapid growth of the MUSIC prostate cancer efforts, the collaborative has expanded to address the care of patients with kidney stones and localized kidney tumors in Michigan. The MUSIC Reducing Operative Complications from Kidney Stones (ROCKS) program was established in an effort to reduce complications among patients undergoing surgery for nephrolithiasis. The program is working towards specific goals of improving patient education, enhancing medical management, improving management of ureteral stents, and increasing physician availability to patients with renal stones. More recently, the MUSIC Kidney program was established with the goal of improving the quality of care for patients with localized renal tumors. This arm of the collaborative is working towards reducing the burden of treatment for patients with small renal masses, avoiding treatment for patients with benign or clinically indolent tumors, and optimizing the evaluation and management strategies for these patients.

The American Board of Urology requires ongoing participation in Outcomes and Quality Improvement as part of the Life Long Learning program, formerly Maintenance of Certification Program, whereby each participating 
urologist is encouraged to track and improve individual outcomes (51). While gaining insight into one's clinical outcomes is critically important, such undertaking may be challenging for individual providers, and participation in data registries, such as MUSIC, AQUA and PURC, may effectively fulfill this requirement.

More generally, urologic QICs have also taken strides in optimizing the efficiency of data abstraction within the collaboratives. At present, data abstraction using trained abstractors is proving to be labor-intensive with a relatively high cost burden. Automated EMR scrubbing, as employed by AQUA, may be more cost-effective but can introduce data inaccuracies (52). Further refinement of techniques for automated data abstraction should improve the accuracy of abstracted data while requiring less manpower than is needed for manual data abstraction.

Finally, the funding of urologic QICs will remain a challenge to be addressed in the future. MUSIC led the way in 2017 by launching the first Collaborative Quality Initiative Value Based Reimbursement (CQIVBR) program in Michigan, with 12 additional specialty QICs to follow (https://www.bcbsm.com/providers/valuepartnerships/physician-group-incentive-prog/specialists/ areas-of-development.html accessed 8/12/19). VBR schedule is updated annually and details objective metrics used to define additional provider payments, incentivizing participation in the registry and promoting high-value care. Examples of prior MUSIC-wide population metrics selected by the collaborative include minimizing lowvalue imaging, optimizing appropriate use of AS and confirmatory testing, and increasing patient enrollment in MUSIC patient reported outcomes (PRO), and achieving these pre-set goals resulted in an additional $3 \%$ payment by BCBSM (James E. Montie, MD, personal communication). Aside from MUSIC's unique partnership with BCBSM, QICs will need to identify other progressive payors, secure federal or state funding and/or develop responsible partnerships with the industry to maintain operations.

In the near future, as pay-for-performance reimbursement models become more prevalent, QICs may become increasingly valuable for their ability to track quality and efficiency outcomes accurately and in real time. Data from QICs will allow practices to more easily identify areas in need of improvement in order to optimize patient care and maximize revenue for the care delivered.

\section{Conclusions}

Urologic QICs are effective in identifying actionable data regarding practice patterns and patient outcomes on a regional and national level. Established QICs have proven to be successful in suggesting and implementing practice change, resulting in improved quality, safety and value of care. The initial experience of these collaboratives has shown that strong physician leadership, coordinating center support, and engaged clinical champions across practice sites are essential to assure success. Although a regional scale may allow for easier in-person collaboration and more rapid implementation of directives, QICs also have great potential to create significant value on a national level. With the initial success of these quality collaboratives in addressing prostate cancer outcomes, the field is poised for expansion of these efforts to potentially address all aspects of urologic disease.

\section{Acknowledgments}

Funding: None.

\section{Footnote}

Provenance and Peer Review: This article was commissioned by the Guest Editors (Marc C. Smaldone and Jeffrey J. Tomaszewski) for the series "Controversies in Minimally Invasive Urologic Oncology" published in Translational Andrology and Urology. The article has undergone external peer review.

Conflicts of Interest: Both authors have completed the ICMJE uniform disclosure form (available at http://dx.doi. org/10.21037/tau.2019.10.18). The series "Controversies in Minimally Invasive Urologic Oncology” was commissioned by the editorial office without any funding or sponsorship. The authors have no other conflicts of interest to declare.

Ethical Statement: The authors are accountable for all aspects of the work in ensuring that questions related to the accuracy or integrity of any part of the work are appropriately investigated and resolved.

Open Access Statement: This is an Open Access article distributed in accordance with the Creative Commons Attribution-NonCommercial-NoDerivs 4.0 International License (CC BY-NC-ND 4.0), which permits the noncommercial replication and distribution of the article with the strict proviso that no changes or edits are made and the original work is properly cited (including links to both the 
formal publication through the relevant DOI and the license). See: https://creativecommons.org/licenses/by-nc-nd/4.0/.

\section{References}

1. Martin $\mathrm{AB}$, Hartman $\mathrm{M}$, Washington $\mathrm{B}$, et al. National Health Care Spending In 2017: Growth Slows To PostGreat Recession Rates; Share Of GDP Stabilizes. Health Aff (Millwood) 2019;38:101377hlthaff201805085.

2. Ghaferi AA. Surgical Collaboratives for Quality Improvement. Surg Oncol Clin N Am 2018;27:633-40.

3. Auffenberg GB, Ghani KR, Ye Z, et al. Comparing Publicly Reported Surgical Outcomes With Quality Measures From a Statewide Improvement Collaborative. JAMA Surg 2016;151:680-2.

4. Hicks CW, Makary MA. A prophet to modern medicine: Ernest Amory Codman. BMJ 2013;347:f7368.

5. In To Err is Human: Building a Safer Health System. Kohn LT, Corrigan JM, Donaldson MS, Editors. Washington (DC), 2000.

6. McCarthy M. Controversial online "scorecard" shows complication rates of 17,000 US surgeons. BMJ 2015;351:h3873.

7. Kaye DR, Norton EC, Ellimoottil C, et al. Understanding the relationship between the Centers for Medicare and Medicaid Services' Hospital Compare star rating, surgical case volume, and short-term outcomes after major cancer surgery. Cancer 2017;123:4259-67.

8. Campbell DA Jr, Kubus JJ, Henke PK, et al. The Michigan Surgical Quality Collaborative: a legacy of Shukri Khuri. Am J Surg 2009;198:S49-55.

9. Luckenbaugh AN, Miller DC, Ghani KR. Collaborative quality improvement. Curr Opin Urol 2017;27:395-401.

10. Birkmeyer JD, Dimick JB, Birkmeyer NJ. Measuring the quality of surgical care: structure, process, or outcomes? J Am Coll Surg 2004;198:626-32.

11. Montie JE. Quality of care in urology. BJU Int 2004;94:755-6.

12. Yu J, Massarweh NN. Surgical Quality Improvement: Working Toward Value or a Work in Progress? J Surg Res 2019;235:160-6.

13. O'Connor GT, Plume SK, Olmstead EM, et al. A regional intervention to improve the hospital mortality associated with coronary artery bypass graft surgery. The Northern New England Cardiovascular Disease Study Group. JAMA 1996;275:841-6.

14. Khuri SF, Daley J, Henderson W, et al. The Department of Veterans Affairs' NSQIP: the first national, validated, outcome-based, risk-adjusted, and peer-controlled program for the measurement and enhancement of the quality of surgical care. National VA Surgical Quality Improvement Program. Ann Surg 1998;228:491-507.

15. Shiloach M, Frencher SK Jr, Steeger JE, et al. Toward robust information: data quality and inter-rater reliability in the American College of Surgeons National Surgical Quality Improvement Program. J Am Coll Surg 2010;210:6-16.

16. Etzioni DA, Wasif N, Dueck AC, et al. Association of hospital participation in a surgical outcomes monitoring program with inpatient complications and mortality. JAMA 2015;313:505-11.

17. Nikolian VC, Regenbogen SE. Statewide Clinic Registries: The Michigan Surgical Quality Collaborative. Clin Colon Rectal Surg 2019;32:16-24.

18. Share DA, Campbell DA, Birkmeyer N, et al. How a regional collaborative of hospitals and physicians in Michigan cut costs and improved the quality of care. Health Aff (Millwood) 2011;30:636-45.

19. Burks FN, Liu AB, Suh RS, et al. Understanding the use of immediate intravesical chemotherapy for patients with bladder cancer. J Urol 2012;188:2108-13.

20. Miller DC, Murtagh DS, Suh RS, et al. Regional collaboration to improve radiographic staging practices among men with early stage prostate cancer. J Urol 2011;186:844-9.

21. askMUSIC website. [Website] [cited 2019 3/28/2019]. Available online: http://musicurology.com/faqs/

22. Auffenberg GB, Ghani KR, Ramani S, et al. askMUSIC: Leveraging a Clinical Registry to Develop a New Machine Learning Model to Inform Patients of Prostate Cancer Treatments Chosen by Similar Men. Eur Urol 2019;75:901-7.

23. Riedinger CB, Womble PR, Linsell SM, et al. Variation in prostate cancer detection rates in a statewide quality improvement collaborative. J Urol 2014;192:373-8.

24. Womble PR, Montie JE, Ye Z, et al. Contemporary use of initial active surveillance among men in Michigan with low-risk prostate cancer. Eur Urol 2015;67:44-50.

25. American Urological Association, Choosing Wisely: Five Things Physicians and Patients Should Question. 2017 [cited 2019 March 17, 2020]. Available online: https:// www.choosingwisely.org/societies/american-urologicalassociation/

26. Merdan S, Womble PR, Miller DC, et al. Toward better use of bone scans among men with early-stage prostate cancer. Urology 2014;84:793-8.

27. Risko R, Merdan S, Womble PR, et al. Clinical predictors and recommendations for staging computed tomography 
scan among men with prostate cancer. Urology

2014;84:1329-34.

28. Hurley P, Dhir A, Gao Y, et al. A Statewide Intervention Improves Appropriate Imaging in Localized Prostate Cancer. J Urol 2017;197:1222-8.

29. Ross I, Womble P, Ye J, et al. MUSIC: patterns of care in the radiographic staging of men with newly diagnosed low risk prostate cancer. J Urol 2015;193:1159-62.

30. Borghesi M, Ahmed H, Nam R, et al. Complications After Systematic, Random, and Image-guided Prostate Biopsy. Eur Urol 2017;71:353-65.

31. Womble PR, Dixon MW, Linsell SM, et al. Infection related hospitalizations after prostate biopsy in a statewide quality improvement collaborative. J Urol 2014;191:1787-92.

32. Womble PR, Linsell SM, Gao Y, et al. A Statewide Intervention to Reduce Hospitalizations after Prostate Biopsy. J Urol 2015;194:403-9.

33. Bianco FJ Jr, Riedel ER, Begg CB, et al. Variations among high volume surgeons in the rate of complications after radical prostatectomy: further evidence that technique matters. J Urol 2005;173:2099-103.

34. Hartz A, He T, Strope S, et al. Surgeon variation in patient quality of life after radical prostatectomy. J Urol 2013;189:1295-301.

35. Myers SN, Ghani KR, Dunn RL, et al. Notable Outcomes and Trackable Events after Surgery: Evaluating an Uncomplicated Recovery after Radical Prostatectomy. J Urol 2016;196:399-404.

36. Auffenberg GB, Lane BR, Linsell S, et al. Practice- vs Physician-Level Variation in Use of Active Surveillance for Men With Low-Risk Prostate Cancer: Implications for Collaborative Quality Improvement. JAMA Surg 2017;152:978-80.

37. Botejue M, Abbott D, Danella J, et al. Utilization of Active Surveillance as Initial Management of Newly Diagnosed Prostate Cancer: Data from the PURC. J Urol 2019;201:929-936.

38. Auffenberg GB, Lane BR, Linsell S, et al. A Roadmap for Improving the Management of Favorable Risk Prostate Cancer. J Urol 2017;198:1220-2.

39. Ginzburg S, Reese A, Trabulsi E, et al. MP08-16 Use of duplicate axial imaging in newly diagnosed prostate cancer - trends across the pennsylvania urologic regional collaborative (PURC). J Urol 2017;197:e97-8.

40. Ginzburg S, Trabulsi E, Reese A, et al. MP14-19 Correlation of multiparametric mri findings and final prostatectomy pathology across the pennsylvania urologic regional collaborative (PURC). J Urol 2018;199:e188.
41. Raman J D, Smaldone M, Guzzo T, et al. MP51-18 Practice patterns of prostate biopsy antibiotic prophylaxis in over 4000 patients from the pennsylvania urologic regional collaboration (PURC). J Urol 2018;199:e692.

42. Botejue M, Reese A, Abbott D, et al. PD20-09 Racial variation in use of active surveillance for the management of low risk prostate cancer in a regional collaborative. J Urol 2018;199:e405.

43. AUA Quality Registry (AQUA) Program Information. [cited 2019 March 20, 2019]. Available online: https:// www.auanet.org/practice-resources/aua-quality-(aqua)registry/program-information

44. Hayes MC, Breen DJ. Excision Versus Ablation in Renal Cancer: Optimising Outcome and Minimising Risk. Eur Urol 2016;69:683-4.

45. Shelton J, Pichardo D, Meeks W, et al. LBA1 Characteristics of participants in the aua quality (aqua) registry and early impact of participation on quality of care. J Urol 2018;199:e576-7.

46. Cooperberg M, Fang R, Wolf J JS, et al. PNFBA-07 The current management of prostate cancer in the united states: data from the aqua registry. J Urol 2017;197:e911-2.

47. A UA. Abstracts of the Mid-Atlantic Section of the American Urological Association Annual Meeting October 4-7, 2018 Washington, DC. Can J Urol 2018;25:9534-67.

48. Spratt DE, Dai DLY, Den RB, et al. Performance of a Prostate Cancer Genomic Classifier in Predicting Metastasis in Men with Prostate-specific Antigen Persistence Postprostatectomy. Eur Urol 2018;74:107-14.

49. Morgan TM, Dunn R, Susan L, et al. Prospective randomized trial of genomic classifier impact on treatment decisions in patients at high risk of recurrence following radical prostatectomy (G-MINOR). J Clin Oncol 2018;36:TPS154.

50. Patel HD, Srivastava A, Patel ND, et al. A Prospective Cohort Study of Postdischarge Opioid Practices After Radical Prostatectomy: The ORIOLES Initiative. Eur Urol 2019;75:215-8.

51. Ritchey ML. Overview: MOC/Life Long Learning Update. Available online: http://www.abu.org/lifelonglearning/overview/. Accessed 8/12/19

52. Tyson MD, Barocas DA. Improving quality through clinical registries in urology. Curr Opin Urol 2017;27:375-9.

Cite this article as: Reese AC, Ginzburg S. The past, present, and future of urological quality improvement collaboratives. Transl Androl Urol 2021;10(5):2280-2288. doi: 10.21037/ tau.2019.10.18 